1 Title: Elevation of FAM129A in neutrophils exposed to serum of patients with

2 severe sepsis: in silico investigations during a hands on training workshop and

3 follow on validation of protein expression in neutrophils.

4

5 Authors: Jessica Roelands ${ }^{1}$, Laurent Chiche ${ }^{2}$, Radu Marches ${ }^{3}$, Mohammed Toufiq ${ }^{1}$,

6 Basirudeen Ahamed Kabeer ${ }^{1}$, Mohamed Alkhair Ibrahim Alfaki ${ }^{1}$, Marwa Saadaoui ${ }^{1}$,

7 Arun Prasath Lakshmanan ${ }^{1}$, Dhinoth Kumar Bangarusamy ${ }^{1}$, Selvasankar Murugesan ${ }^{1}$,

8 Davide Bedognetti ${ }^{1}$, Wouter Hendrickx $^{1}$, Souhaila Al Khodor ${ }^{1}$, Annalisa Terranegra ${ }^{1}$,

9 Jacques Banchereau ${ }^{3}$, Mathieu Garand ${ }^{1}$, Damien Chaussabel ${ }^{1}$, and Darawan Rinchai ${ }^{1}$,

\title{
11 Affiliation:
}

121 Sidra Medicine, Doha, Qatar;

132 Hopital Europeen, Marseille, France,

143 Jackson Laboratory for Genomic Medicine, Farmington, CT, USA

15

16

17 * To whom correspondence may be addressed: drinchai@sidra.org 


\section{ABSTRACT}

19 Steps involved in reductionist investigation approaches can be imitated using public

20 transcriptome datasets as source of training material. In the present report trainees

21 explored an apparent gap in biological knowledge for FAM129A (family with sequence

22 similarity 129 member A). Elevated abundance of FAM129A transcripts were observed in

23 a transcriptome dataset where neutrophils were exposed in vitro to plasma of patients

24 with sepsis. However, no literature linking FAM129A and either neutrophils, sepsis or

25 inflammation could be identified. Additional datasets were selected to independently

26 validate this initial observation and further explore differential expression of FAM129A in

27 the context of sepsis studies. Follow on investigations carried out at the bench confirmed

28 restriction of the expression of FAM129A protein at the surface of circulating blood

29 neutrophils and monocytes. A potential role for FAM129A in neutrophil survival was

30 inferred from profiling of literature associated with FAM129A, which remains to be

31 investigated in further follow on investigations. 


\section{INTRODUCTION}

Sepsis is a life-threatening condition characterized by multiple organ dysfunction [1]. Understanding of the underlying pathogenic mechanism has greatly improved over the past several years. Yet interventions aiming at modulating the exaggerated immune response to infection, one of the root causes of sepsis complications, are yet to

52 materialize in clinical practice. Neutrophils constitute a first line of defense against bacterial infections. This specialized population of leukocytes carries effector functions

54 via the production of immune mediators as well as through microbicidal activity, either 55 resulting from degranulation or through a process that is referred to as "netosis" which consists in formation of extracellular traps that serve to immobilize and kill invading 57 pathogens [2].

In earlier work we have demonstrated the singular ability of neutrophils to respond

59 to immunomodulatory factors present in the plasma of patients with severe sepsis ([3] / GSE49758). This transcriptome dataset which at the time was deposited in the NCBI's gene expression omnibus was employed more recently in the context of a training workshop conducted at Sidra Medicine in Doha Qatar in November of 2018. Trainees

63 applied reductionist investigation approaches, using available public omics data as a 64 starting point (COD1 training module, as described in: [4]). Individual reports from trainees 65 are included with this submission as supplementary files. Potential gaps in biomedical 66 knowledge were assessed as a first step. It was thus determined that expression of 67 FAM129A by neutrophils had not been reported in the biomedical literature and neither 68 had its induction under inflammatory conditions or in the context of sepsis. The next step 69 consisted in confirming the initial observation in independent datasets and inferring from 
70 the literature a potential role for FAM129A in the context of sepsis, inflammation and/or

71 neutrophil immunobiology. Experiments were in addition performed to confirm expression

72 of FAM129A protein in neutrophils.

\section{MATERIALS AND METHODS}

\section{Workshop format}

Briefly, a workshop was organized in November of 2018 at Sidra Medicine in Doha,

Qatar. It consisted in applying reductionist investigation approaches while using public

80 transcriptome data as source of training material (COD1 training module [4]). Trainees

81 who joined the workshop carried out hands on activities focusing on two candidate genes,

82 ANXA3 and FAM129A. For each candidate gene activities were guided by two

83 experienced instructors. Manuscript preparation was the primary responsibility of the

84 instructors and of the senior investigator leading the program. Workshop participants

85 prepared each a report which are made available as part of this publication as

86 supplementary files. More details regarding the COD1 training module, including

87 educational material shared during this workshop, will be made available via a separate

88 publication.

89

\section{Access to reference datasets}

91 The primary dataset employed as a starting point for identification of gene

92 candidates was deposited in GEO by Khaenam et al ([3], accession GSE49758). This

93 and other reference public transcriptome datasets relevant to sepsis, neutrophil

94 immunobiology or inflammation were deposited in a data browsing application (GXB:

95 http://sepsis.gxbsidra.org/dm3/geneBrowser/list). A total of 48 datasets were included in 
96 this curated collection. Each dataset can be individually accessed and profiles of any

97 transcript measure on the array plotted interactively. The GXB tool has been described in

98 details in an earlier publication [5]. Constitution of curated dataset collections is an activity

99 that is undertaken as part of the COD2 training module, as described in a recent review $100 \quad[4]$.

\section{RESULTS}

105

106

108

\section{FAM129A levels are increased in neutrophils exposed to septic serum}

The "primary" dataset used as a basis for identification of candidate genes was generated and deposited in GEO by our team in the context of an earlier publication [3]. In this experiment neutrophils were isolated from the blood of healthy individuals and exposed in vitro for 6 hours to plasma from adult patients with sepsis. The neutrophil transcriptional response was measured using Illumina beadarrays. FAM129A was prioritized as a target for this training activity on the basis of: 1) increased abundance of this transcripts following in vitro exposure of neutrophils to serum of septic patients (GSE49758, [3]), and 2) the extent of the overlap between the FAM129A literature and literature on sepsis, neutrophil or inflammation. Figure 1 shows levels of FAM129A transcript abundance in neutrophils exposed to plasma from subjects with non-severe or 


\section{Knowledge gap assessment}

The next assignment consisted in assessing the extent of the current biomedical

123 knowledge regarding the role of FAM129A in the context of sepsis, inflammation or

124 neutrophil immunobiology. First, a PubMed query was designed that would retreive the

125 FAM129A literature. Official symbol, name and aliases available from the NCBI Entrez

126 Gene database were combined in the following query:

"sequence similarity 129 member A" [tw] OR FAM129A [tw] OR C1orf24 [tw] OR

128 NIBAN [tw]

As of January of 2019 , a list of 35 articles was returned when running this PubMed

130 query (listed in Supplementary file 1). Next, links between the FAM129A and

131 inflammation, neutrophil or sepsis literature were investigated. The Boolean operator

132 AND was added to the FAM129A query followed by the keywords "Inflammation",

133 "Neutrophils" or "Sepsis" (Figure 2). No articles among the FAM129A literature

134 intersected with either the neutrophil or sepsis literature. But one of the 35 articles

135 constituting the FAM129A literature intersected with the inflammation literature [6]. In this

136 study FAM129A was identified as one of four genes associated with airway hyper-

137 responsiveness and differentially expressed in laser microdisected airway smooth muscle

138 cells of asthmatic patients vs non-asthmatic controls. The work did not associate

139 FAM129A with neutrophils or sepsis and did not elaborate on the role of FAM129A in the

140 context of atopy or asthma. 


\section{Independent validation in silico}

The third task assigned to the trainees was to validate the initial finding in

146 independent public datasets. GEO datasets relevant to sepsis or neutrophil

147 immunobiology were curated and uploaded on the custom GXB data browsing

148 application. This resource comprises 48 datasets and is available via this link:

149 http://sepsis.gxbsidra.org/dm3/landing.gsp. This GXB instance was created expressly in

150 support of the activities conducted in the context of workshops utilizing the same primary

151 dataset and pool of candidate genes (as described in a manuscript that is in preparation,

152 GXB has been previously described and is available on GitHub [5]). Trainees were asked

153 to: first select vdatasets that they estimated to be adequate for validation, on the basis of

154 the information about study/experimental design available in GXB or in the linked primary

155 publication, and, secondly, to access the profile of FAM129A in each of the dataset. This

156 two-step process was established to avoid biasing the validation process. In the case of

157 FAM129A, seven datasets were first selected in which abundance levels of transcript

158 were measured in whole blood, PBMCs or neutrophils of patients with sepsis (Table 1)

159 [7-13]. FAM129A transcript abundance was not measured in one of the datasets [13].

160 Increase in FAM129A transcript levels in septic patient as compared to controls was found

161 in four of the six remaining datasets (Figure 3). The characteristics of the study

162 populations varied, with three out of six consisting of pediatric subjects and the other three

163 of adults (Figure 4). One study was conducted in Asia, one in Europe and four in North

164 America. Assay characteristics were also different since in four cases samples were run

165 on Illumina beadchips and in two on Affymetrix GeneChips. The datasets in which no

166 increases in levels of FAM129A were not observed were conducted both in adults and in 
167 North America. One of them profiled whole blood and used Illumina arrays, while the other 168 profiled neutrophils and used Affymetrix arrays. In yet another dataset in which profiles 169 were generated via RNA sequencing increase in abundance of FAM129A could be 170 observed in whole blood of septic patients as well as in isolated monocytes but it was not 171 the case in neutrophils in which abundance levels were uniformly higher 172 (http://sepsis.gxbsidra.org/dm3/miniURL/view/OF) [14] / GSE60424.

174 FAM129A protein is expressed at the surface of monocytes and neutrophils 
190 "tissue", "biomolecules", etc... Subsequently, the prevalence of those keywords in the

191 FAM129A literature was determined. Top keyword or phrases included "thyroid

192 carcinoma" (10 articles out of the 35 constituting the FAM129A literature), which after

193 consolidation with related keyword/phrases such as "thyroid cancer", "follicular thyroid 194 neoplasms", "thyroid follicular tumors", "thyroid tumors" yielded a total of 12 entries. Other 195 major concepts associated with FAM129A were "apoptosis" (7 entries), "proliferation" (6 entries) and "renal tumors" OR "renal carcinogenesis" (5 entries).

Next, these top FAM129A-associated concepts were employed in order to establish indirect relationships with the context of the primary study in which differential FAM129A expression was observed. While only one article could be found connecting

200 FAM129A directly to either the sepsis, inflammation or neutrophil literature, many were 201 found which permitted to establish an indirect connection through via "thyroid cancer", 202 apoptosis or proliferation (Figure 6).

The indirect associations established between FAM129A and sepsis, inflammation

204 and the neutrophil literature were next used as a framework to make inferences about the 205 role of FAM129A in this context as is outlined in the discussion.

\section{DISCUSSION}

209 While no literature describes the role of FAM129A in neutrophils and its relation to sepsis,

210 the biological concepts apoptosis and proliferation have previously been linked to

211 FAM129A. Expression of FAM129A has been frequently reported in human cancer,

212 including thyroid carcinoma, head and neck cancer and renal carcinoma [15-23]. In 213 human cancer cells, an anti-apoptotic role for FAM129A has been described [16, 24]. A 
214 similar role for FAM129A could be possible in neutrophils. Neutrophils have a short life

215 span both in the circulation (8-20 hours) and in tissue (1-4 days). The constitutive

216 neutrophil death is a type of apoptotic death and its regulation is essential for neutrophil

217 homeostasis [25]. A prolonged lifespan of neutrophils has been linked to sepsis in patients

218 with burns, traumatic injuries and pneumonia [26-29]. This could be an explanation for

219 the observed upregulation of FAM129A in neutrophils in association with sepsis or

220 increased severity of sepsis. Thus our hypothesis is that the inhibition of neutrophil

221 apoptosis mediated by FAM129A would lead to an extended life span of neutrophils,

222 subsequently causing damage to healthy tissues. Follow on investigations that could

223 permit testing of this hypothesis include the correlation of FAM129A with p53 expression

224 in neutrophils or assessing FAM129A expression in non-apoptotic and apoptotic 225 neutrophils.

226

227 REFERENCES

228 1. van der Poll, T., et al., The immunopathology of sepsis and potential therapeutic 229 targets. Nat Rev Immunol, 2017. 17(7): p. 407-420.

$230 \quad$ 2. Lipinska-Gediga, M., Neutrophils, NETs, NETosis - old or new factors in sepsis

231 and septic shock? Anaesthesiol Intensive Ther, 2017. 49(3): p. 235-240.

232 3. Khaenam, P., et al., A transcriptomic reporter assay employing neutrophils to 233 measure immunogenic activity of septic patients' plasma. J Transl Med, 2014. 12:

234 p. 65.

235 4. Chaussabel, D. and D. Rinchai, Using 'collective omics data' for biomedical 236 research training. Immunology, 2018. 155(1): p. 18-23. 
237 5. Speake, C., et al., An interactive web application for the dissemination of human 238 systems immunology data. J Transl Med, 2015. 13: p. 196.

239 6. Yick, C.Y., et al., Gene expression profiling of laser microdissected airway 240 smooth muscle tissue in asthma and atopy. Allergy, 2014. 69(9): p. 1233-40.

241 7. Ardura, M.I., et al., Enhanced monocyte response and decreased central memory T cells in children with invasive Staphylococcus aureus infections. PLoS One, 2009. 4(5): p. e5446.

8. Banchereau, R., et al., Host immune transcriptional profiles reflect the variability in clinical disease manifestations in patients with Staphylococcus aureus infections. PLoS One, 2012. 7(4): p. e34390.

9. Demaret, J., et al., Marked alterations of neutrophil functions during sepsisinduced immunosuppression. J Leukoc Biol, 2015. 98(6): p. 1081-90.

10. Pankla, R., et al., Genomic transcriptional profiling identifies a candidate blood biomarker signature for the diagnosis of septicemic melioidosis. Genome Biol, 2009. 10(11): p. R127.

11. Parnell, G.P., et al., Identifying key regulatory genes in the whole blood of septic patients to monitor underlying immune dysfunctions. Shock, 2013. 40(3): p. 16674.

12. Smith, C.L., et al., Identification of a human neonatal immune-metabolic network associated with bacterial infection. Nat Commun, 2014. 5: p. 4649.

13. Wynn, J.L., et al., The influence of developmental age on the early transcriptomic response of children with septic shock. Mol Med, 2011. 17(11-12): p. 1146-56.

259 14. Linsley, P.S., et al., Copy number loss of the interferon gene cluster in melanomas is linked to reduced $T$ cell infiltrate and poor patient prognosis. PLoS One, 2014. 9(10): p. e109760. 
262 15. Adachi, H., et al., Niban gene is commonly expressed in the renal tumors: a new candidate marker for renal carcinogenesis. Oncogene, 2004. 23(19): p. 3495500.

16. Carvalheira, G., B.H. Nozima, and J.M. Cerutti, microRNA-106b-mediated downregulation of C1orf24 expression induces apoptosis and suppresses invasion of

17. Carvalheira, G.M., et al., DDIT3, STT3A (ITM1), ARG2 and FAM129A (Niban, thyroid cancer. Oncotarget, 2015. 6(29): p. 28357-70.

18. Ito, S., et al., Frequent expression of Niban in head and neck squamous cell carcinoma and squamous dysplasia. Head Neck, 2010. 32(1): p. 96-103.

19. Majima, S., et al., A novel gene "Niban" upregulated in renal carcinogenesis: cloning by the cDNA-amplified fragment length polymorphism approach. Jpn J

20. Matsumoto, F., et al., A novel tumor marker, Niban, is expressed in subsets of thyroid tumors and Hashimoto's thyroiditis. Hum Pathol, 2006. 37(12): p. 1592600.

21. Nozima, B.H., et al., FAM129A regulates autophagy in thyroid carcinomas in an oncogene-dependent manner. Endocr Relat Cancer, 2019. 26(1): p. 227-238.

22. Patel, M.R., et al., STT3A, C1orf24, TFF3: putative markers for characterization of follicular thyroid neoplasms from fine-needle aspirates. Laryngoscope, 2011.

23. Sigstad, E., et al., The new molecular markers DDIT3, STT3A, ARG2 and FAM129A are not useful in diagnosing thyroid follicular tumors. Mod Pathol, 2012. 25(4): p. 537-47. 
288

24. Ji, H., et al., AKT-dependent phosphorylation of Niban regulates nucleophosminand MDM2-mediated p53 stability and cell apoptosis. EMBO Rep, 2012. 13(6): p. 554-60.

25. Wasyluk, J.S. and Z. Wegrzyn, Problem of objective performance assessment of primary health care physicians. Scand J Prim Health Care, 1991. 9(4): p. 276-80.

26. Altznauer, F., et al., Calpain-1 regulates Bax and subsequent Smac-dependent caspase-3 activation in neutrophil apoptosis. J Biol Chem, 2004. 279(7): p. 594757.

27. Chitnis, D., et al., Inhibition of apoptosis in polymorphonuclear neutrophils from burn patients. J Leukoc Biol, 1996. 59(6): p. 835-9.

28. Ertel, W., et al., Circulating mediators in serum of injured patients with septic complications inhibit neutrophil apoptosis through up-regulation of proteintyrosine phosphorylation. J Trauma, 1998. 44(5): p. 767-75; discussion 775-6.

29. Keel, M., et al., Interleukin-10 counterregulates proinflammatory cytokine-induced inhibition of neutrophil apoptosis during severe sepsis. Blood, 1997. 90(9): p. 3356-63.

\section{ACKNOWLEDGEMENTS}

Sidra Medicine is a member of the Qatar Foundation for Education, Science and Community Development. The work was supported in part via a grant from the Qatar National Research Fund: QNRF NPRP10-0205-170348

\section{AUTHOR CONTRIBUTION}

Conceptualization: JR, DC, DR. Data curation and validation: DR and MT. Visualization: JR, DC, DR. Analysis and interpretation: JR, LC, RM, MT, BAK, MAIA, MS, AL, DKB, MG, 
313 DC, DR. Writing of the first draft: JR, DC, DR. Funding acquisition: DB, DC. Methodology

314 development: MT, BAK, MAIA, MG, DC, DR. Software development and database

315 maintenance: MT. Writing - review \& editing: JR, LC, RM, MT, BAK, MAIA, MS, AL, DKB,

316 DB, WH, SAK, AT, JB, MG, DC, DR. The contributor's roles listed above follow the

317 Contributor Roles Taxonomy (CRediT) managed by The Consortia Advancing Standards

318 in Research Administration Information (CASRAI) (https://casrai.org/credit/).

320 FIGURE LEGENDS

321 FIGURE 1. Abundance of FAM129A transcripts is increased in neutrophils exposed

322 to plasma of severe septic patients. Abundance levels of FAM129A extracted from a

323 dataset by Khaenam et al. with accession GSE49755 are plotted on this figure. It shows

324 a significant increase in FAM129A transcript abundance in neutrophils isolated from

325 healthy subjects and cultured for 6 hours in the presence of plasma from patients with

326 severe sepsis, as compared to levels measured in patients with non-severe sepsis.

328 FIGURE 2. Assessment of gap in FAM129A biomedical knowledge relating to

329 sepsis, inflammation or neutrophil immunobiology. This diagrammatic

330 representation indicates the number of PubMed articles found to be associated with either

331 FAM129A, Neutrophils, Inflammation or Sepsis (each "node", with numbers of articles

332 provided). Numbers provided on edges connecting each of the notes indicate the extent

333 of the overlap. 
335 FIGURE 3: Changes in abundance of FAM129A transcripts in septic patients in six

336 independent datasets. Box plots show levels of FAM129A measured via microarrays in

3376 public datasets. Datasets are arranged in the same order as in Figure 2. In each dataset

338 expression levels between septic patients and control subjects are compared.

340 FIGURE 4: Increase of FAM129A expression in sepsis is observed in four out of six

341 independent datasets. This graph shows fold changes in abundance of FAM129A in six

342 independent datasets selected for follow on investigation of the increase in abundance of

343 FAM129A RNA observed in neutrophils exposed to septic plasma (Figure 1). Each

344 dataset is represented by a spot. The position of the spot on the vertical axis indicates

345 the corresponding fold change in abundance of FAM129A for the dataset. Spots are

346 connected to dataset attributes (metadata). On the top panel attributes relevant to the

347 group comparisons that were performed are shown (groups being compared, whether

348 increase in FAM129A abundance is statistically significant). On the middle and bottom

349 panels attributes describing the study populations and assay, respectively, are overlaid

350 on the same graph. For reference, GEO IDs for the datasets shown on the graph from

351 top to bottom are: GSE13015, GSE16129, GSE25504, GSE30119, GSE64457,

352 GSE54514 (also listed in Table 1). FAM129A transcript abundance profiles for each of

353 these datasets is plotted in Figure 3.

355 FIGURE 5: Flow cytometry profiling of FAM129A protein expression on blood

356 leukocyte populations. The top panel describes the gating strategy, which employed a 357 combination of forward and side scatter measurements along with cell surface markers 
358 CD3, CD14, CD4, CD8, CD11B and CD19 in order to identify peripheral blood

359 mononuclear cell and neutrophil populations in whole blood. The bottom panel shows

360 levels of expression of FAM129A in neutrophils, monocytes (CD14), B-cells (CD19) as

361 well as CD4 and CD8 T-cells.

362

363 FIGURE 6: Identification of concepts indirectly linking the FAM129A literature and

364 literature associated with Neutrophils, Inflammation or Sepsis. Each panel indicates

365 the extent of indirect associations established through one of the top concepts associated

366 with the FAM129A literature. In the top panel this concept is "Thyroid carcinoma". The

367 Pubmed query retrieving the 12 articles in the FAM129A literature in which thyroid

368 carcinoma is mentioned is shown on top of the left most circle. In turn, the query retrieving

36947 articles of the neutrophil literature in which thyroid carcinoma is mentioned is shown

370 on the right of this panel. Similar queries were run to retrieve inflammation or sepsis

371 literature mentioning thyroid carcinoma. The middle and bottom panels are organized

372 similarly but explore respectively apoptosis and inflammation as intermediate concepts.

373

374 TABLES

375 Table 1: Validation datasets used to assess levels of FAM129A RNA abundance in 376 septic patients 


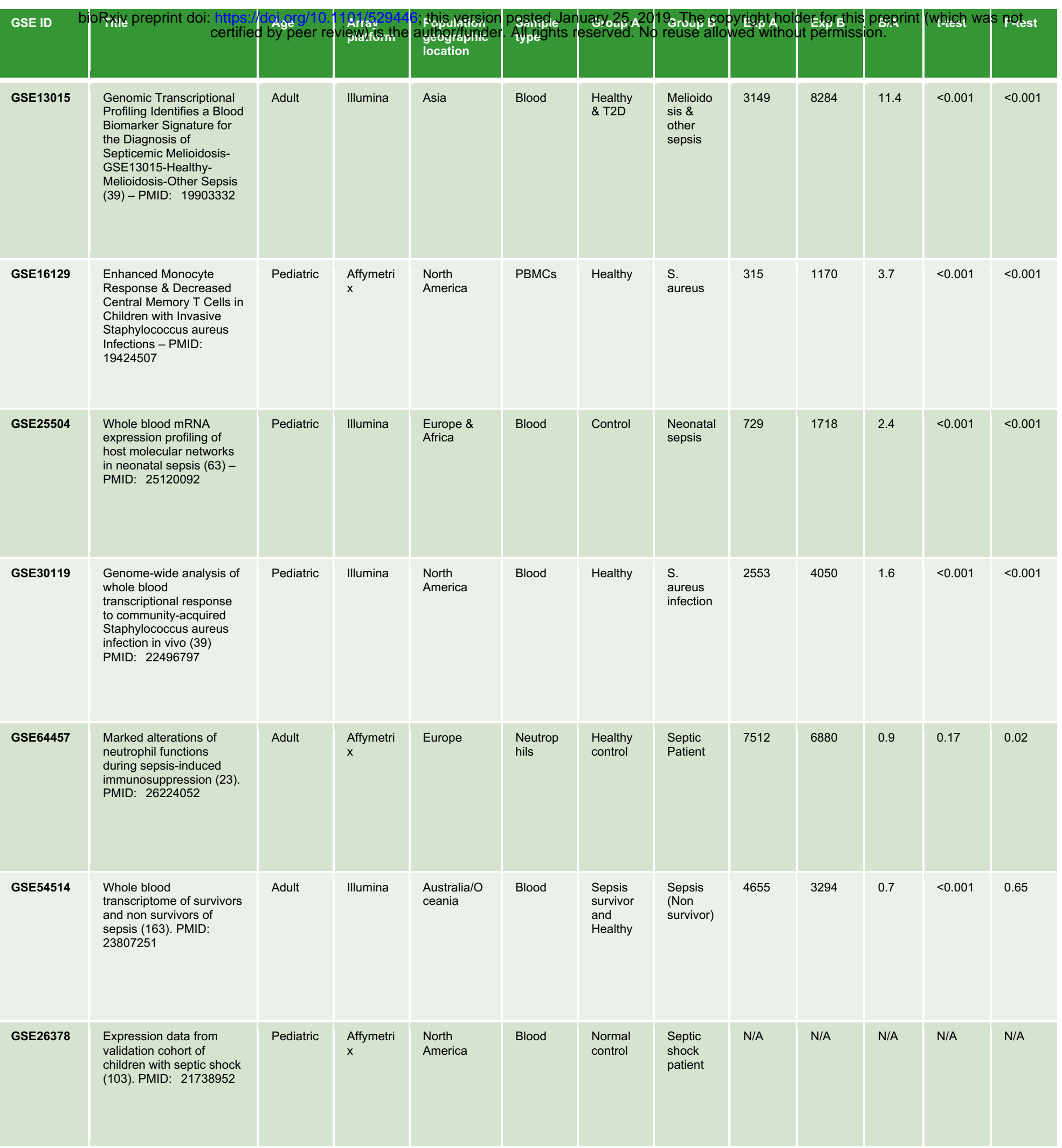




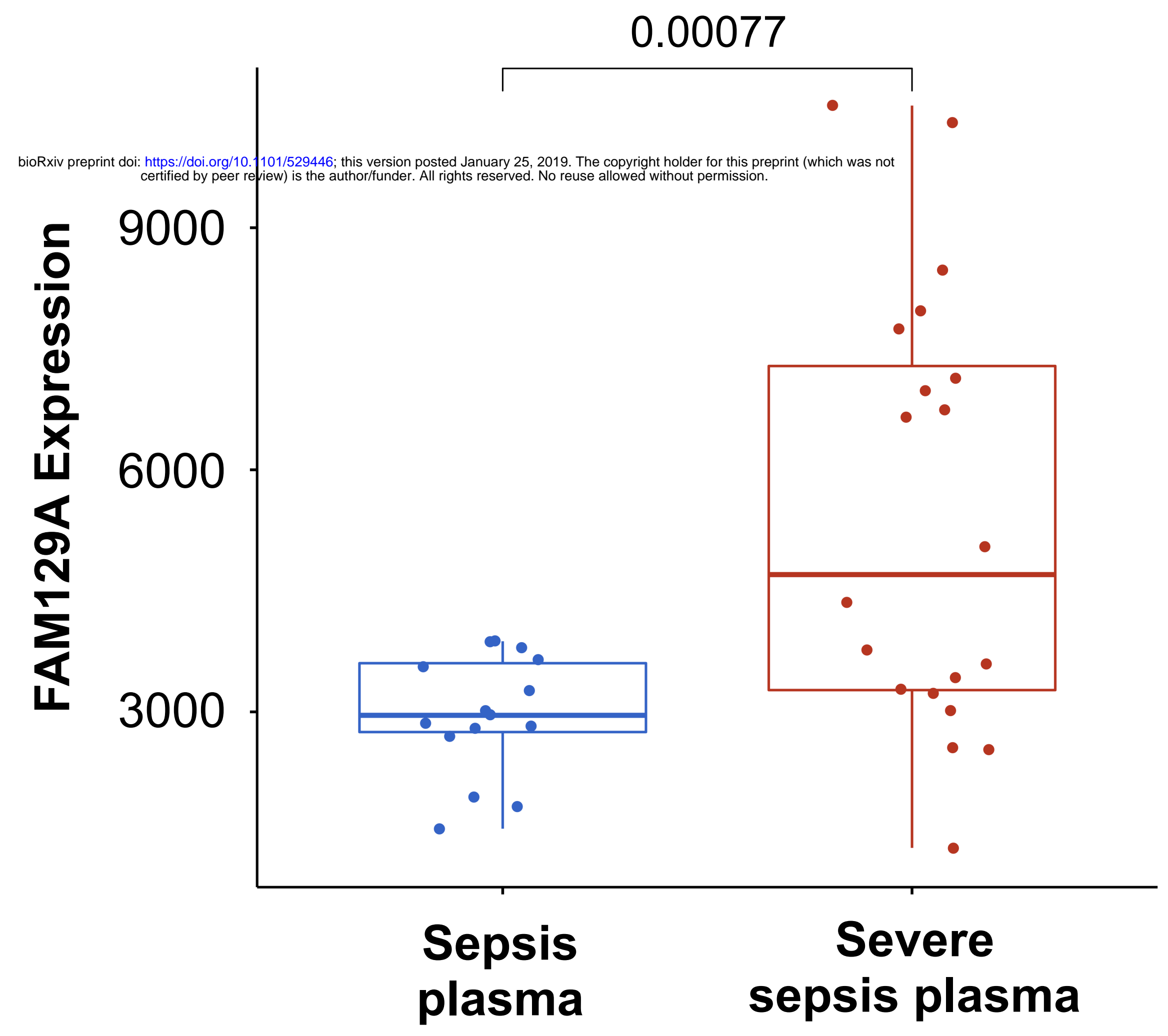

Figure 1 


\section{Neutrophils}

\section{7}

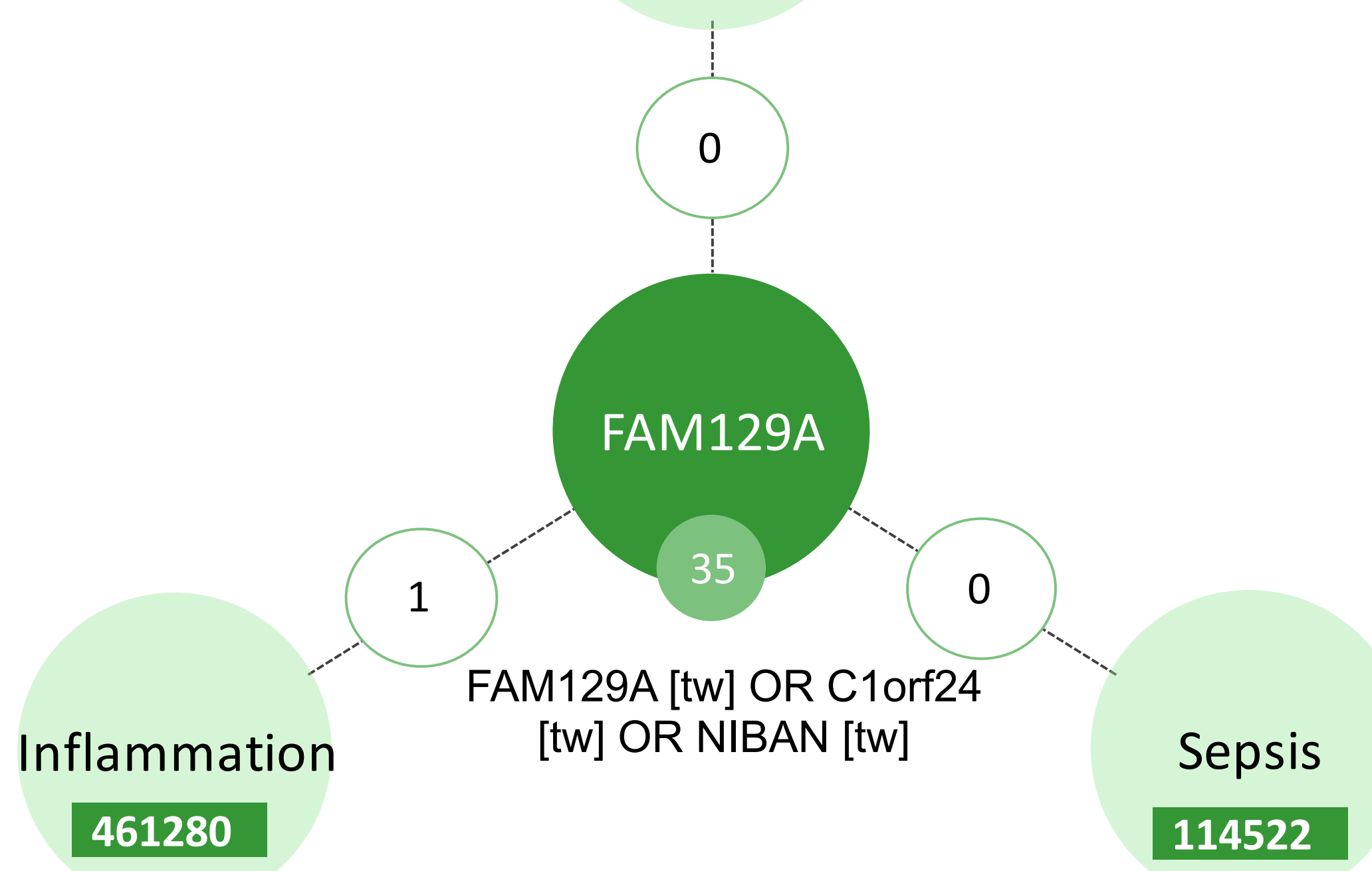

Figure 2 


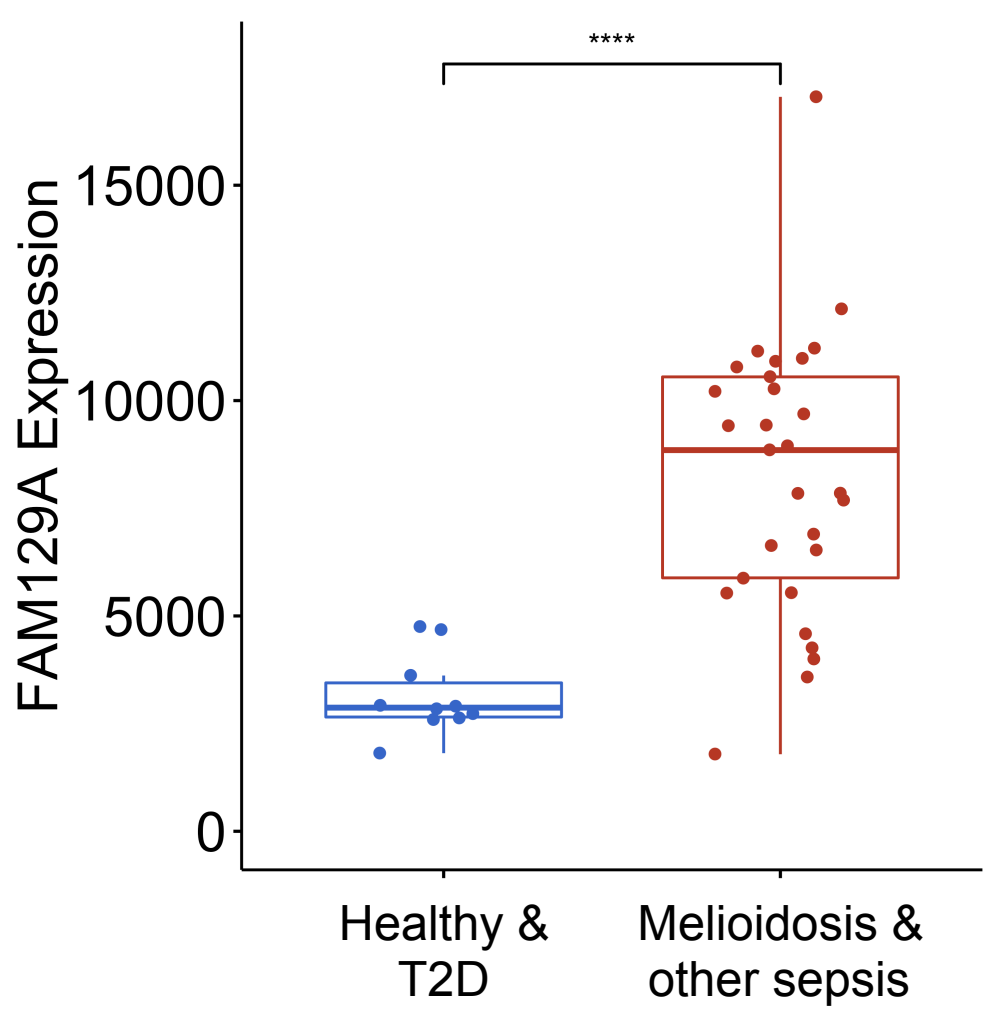

GSE25504

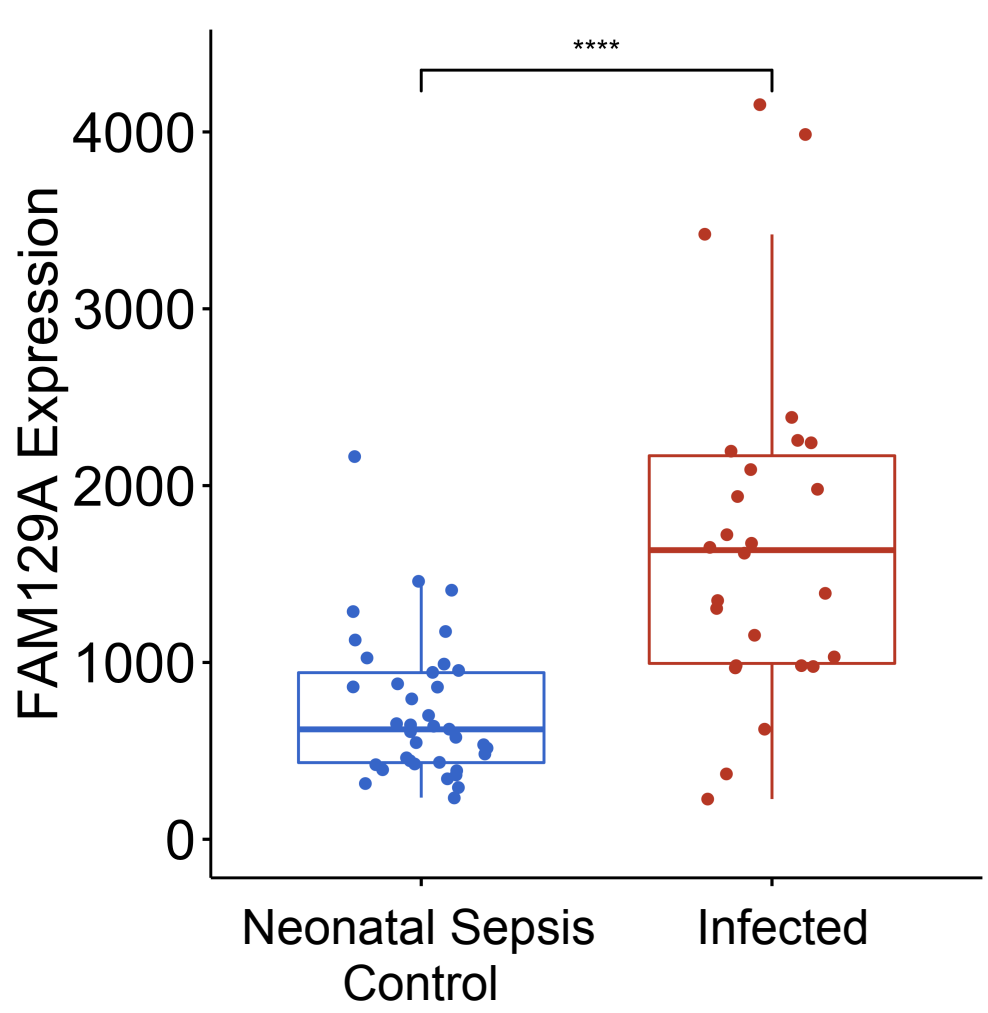

GSE64457

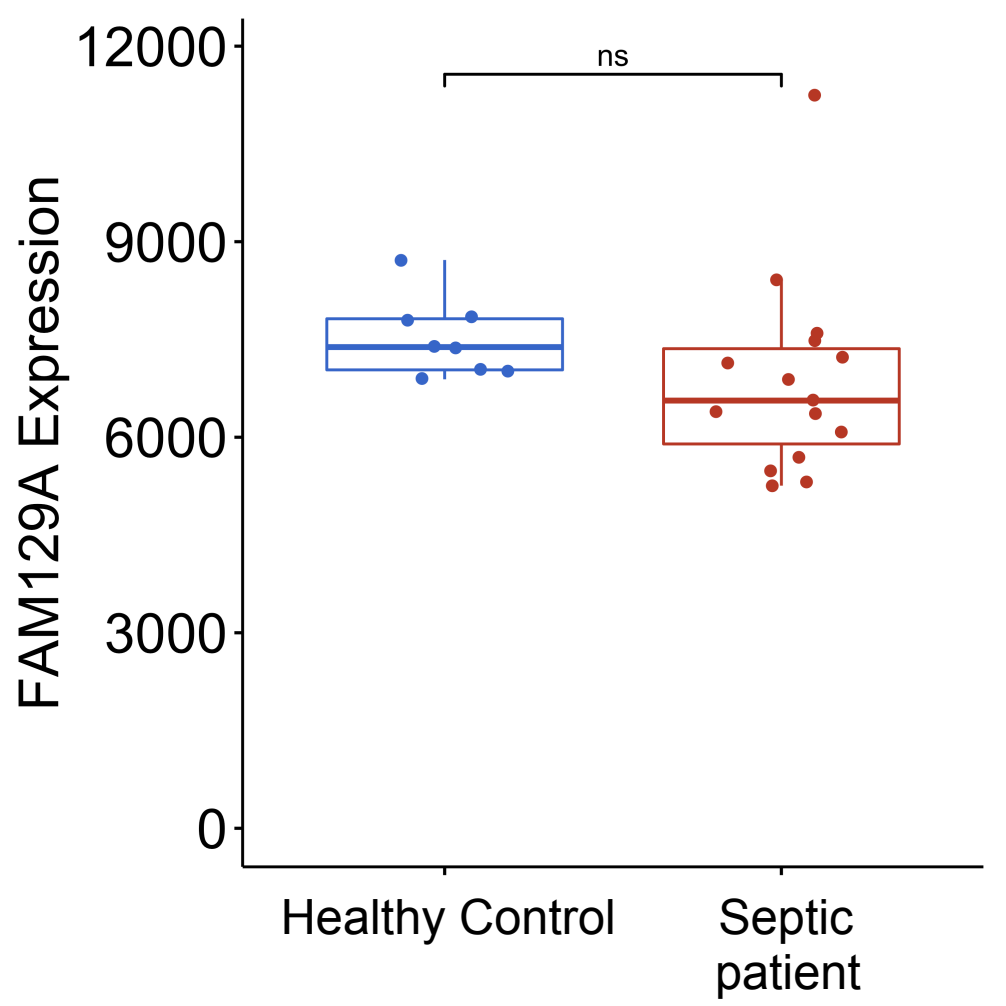

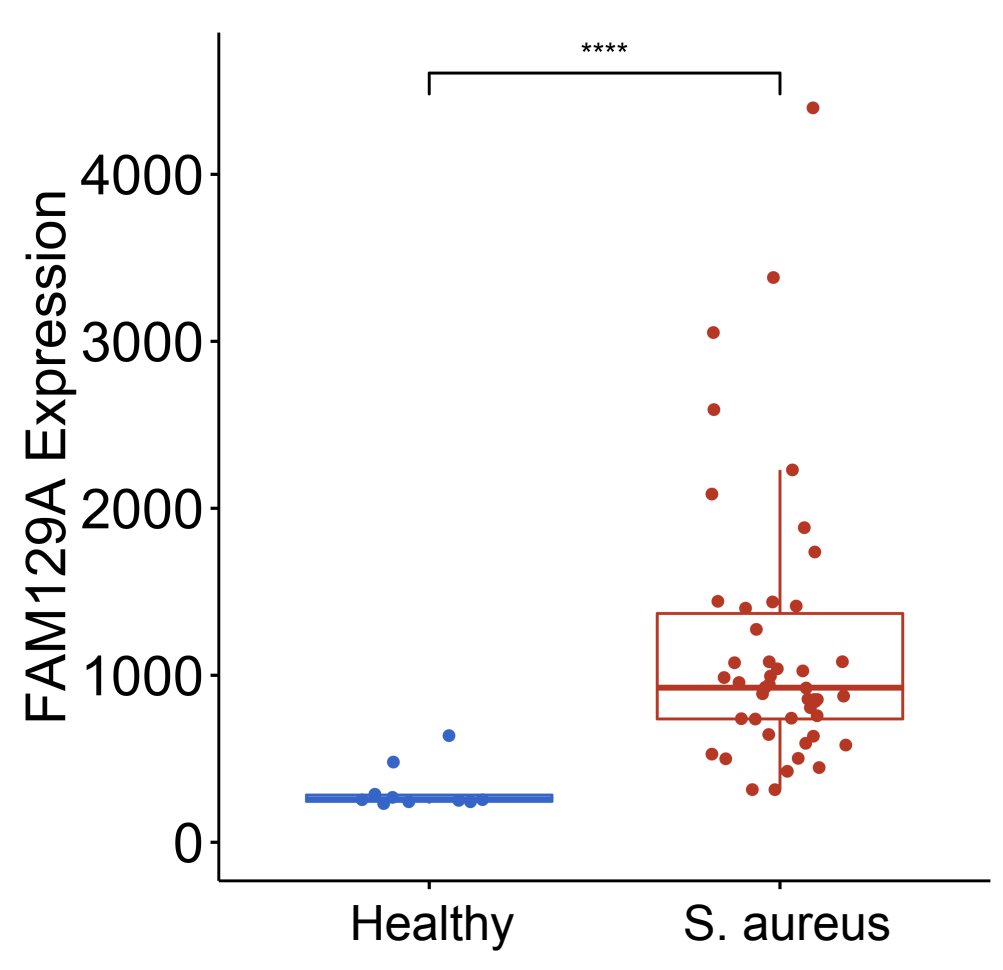

GSE30119

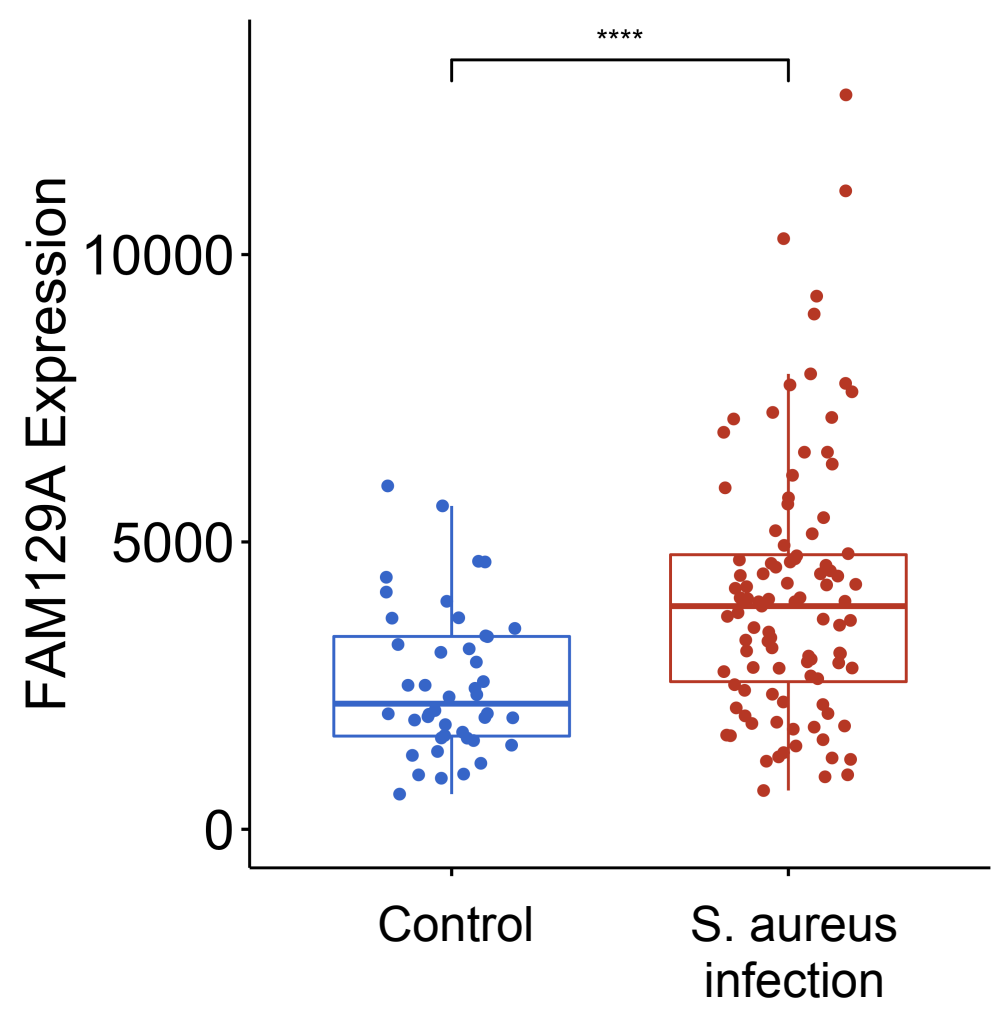

GSE54514

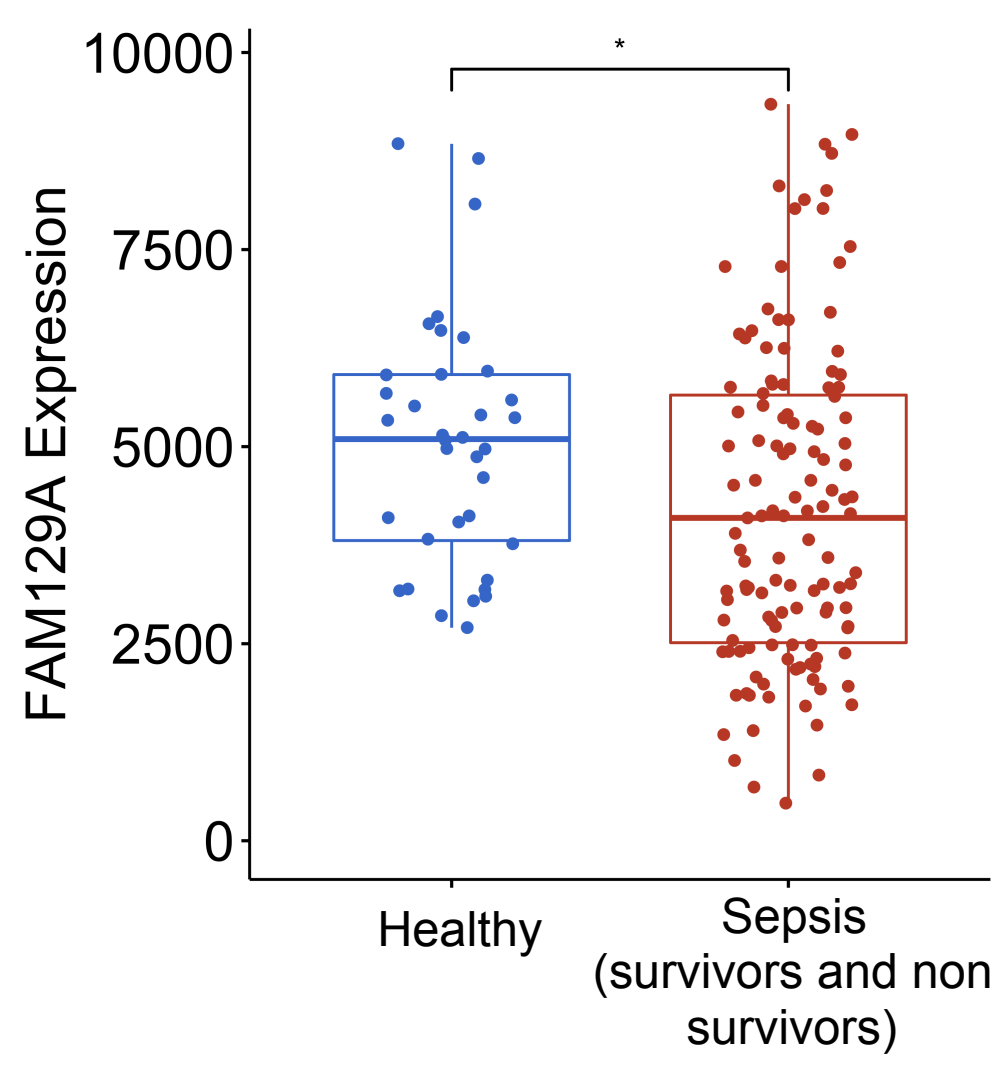

\section{Figure 3}


Comparisons

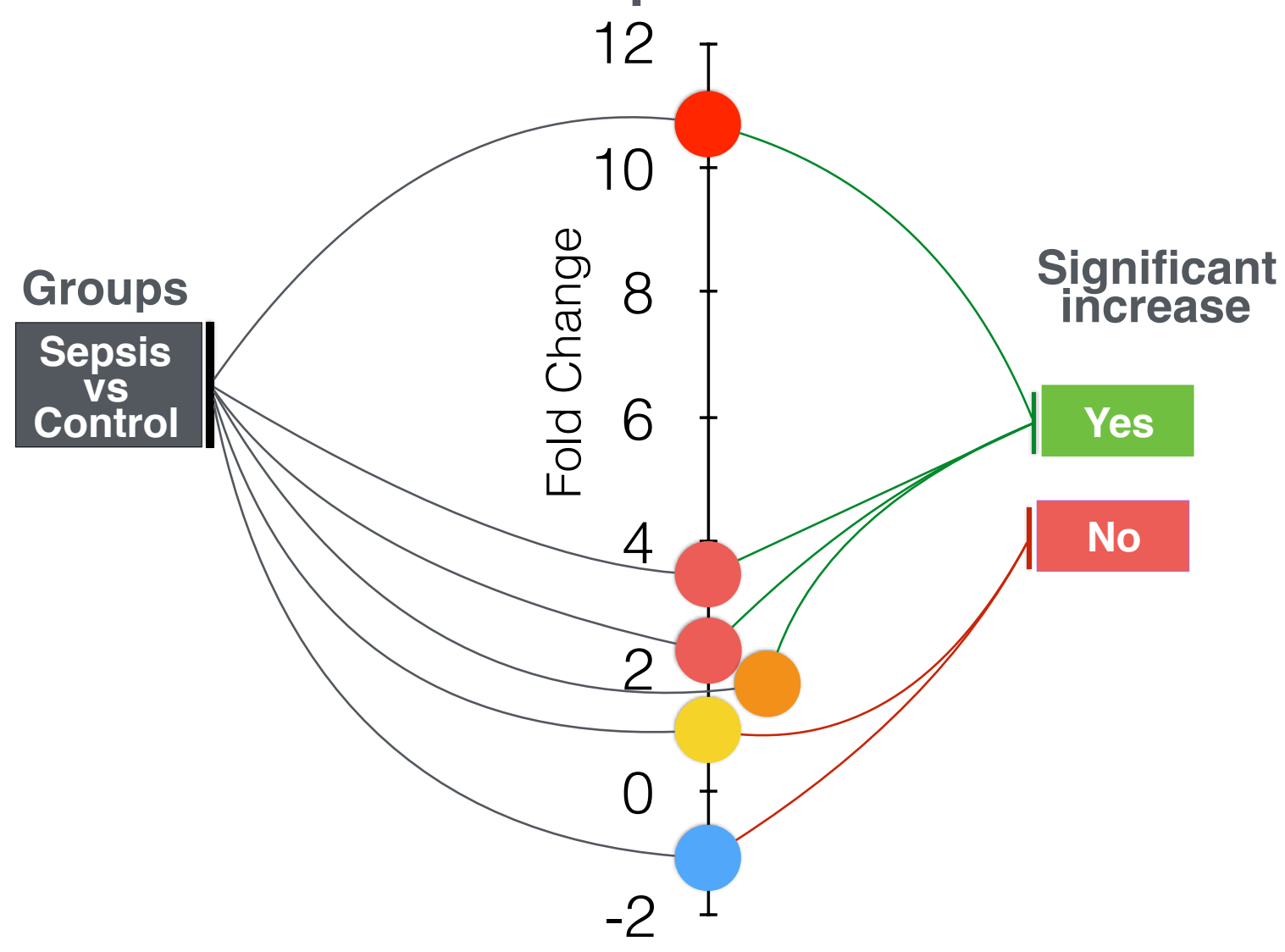

Study populations
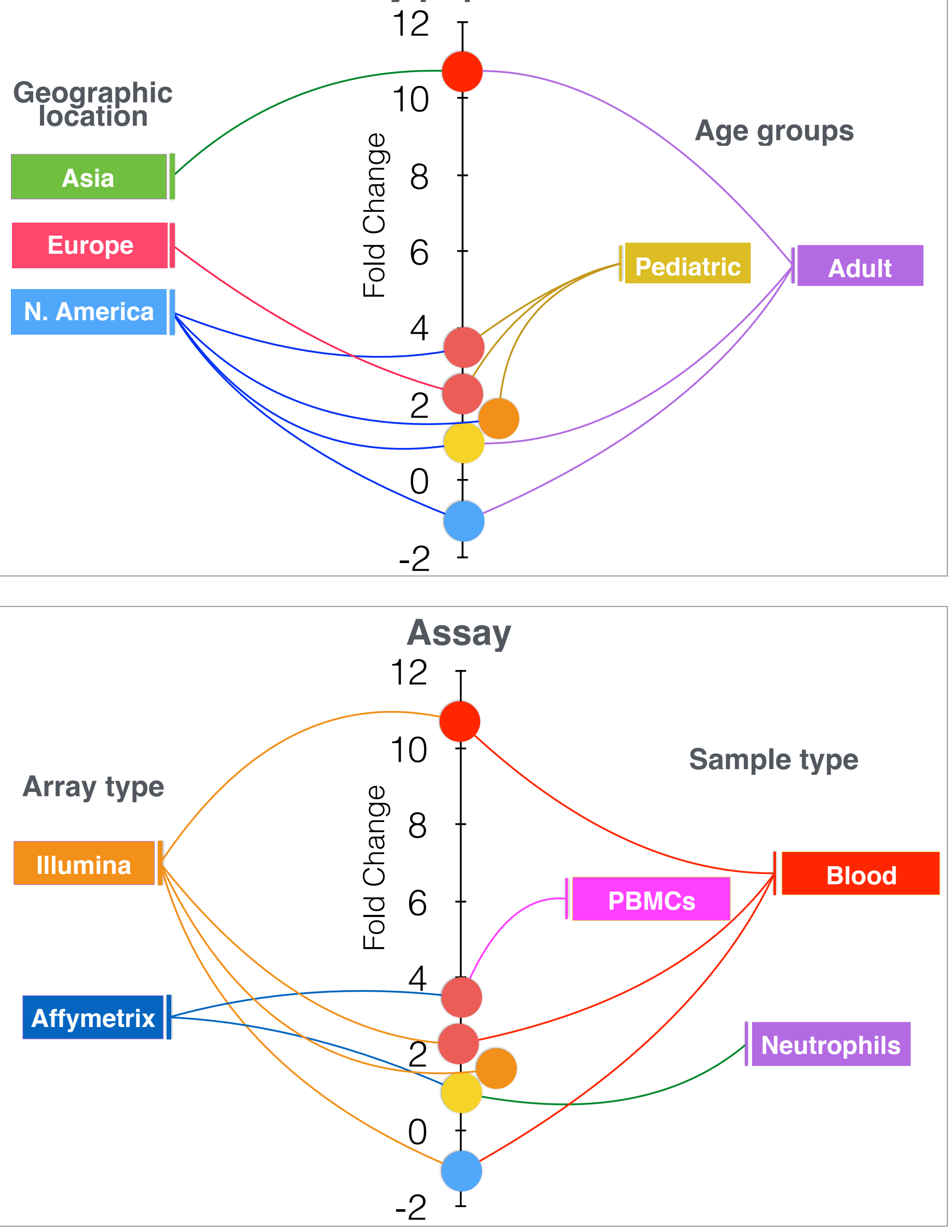

Figure 4 


\section{PBMC Gating Strategy}

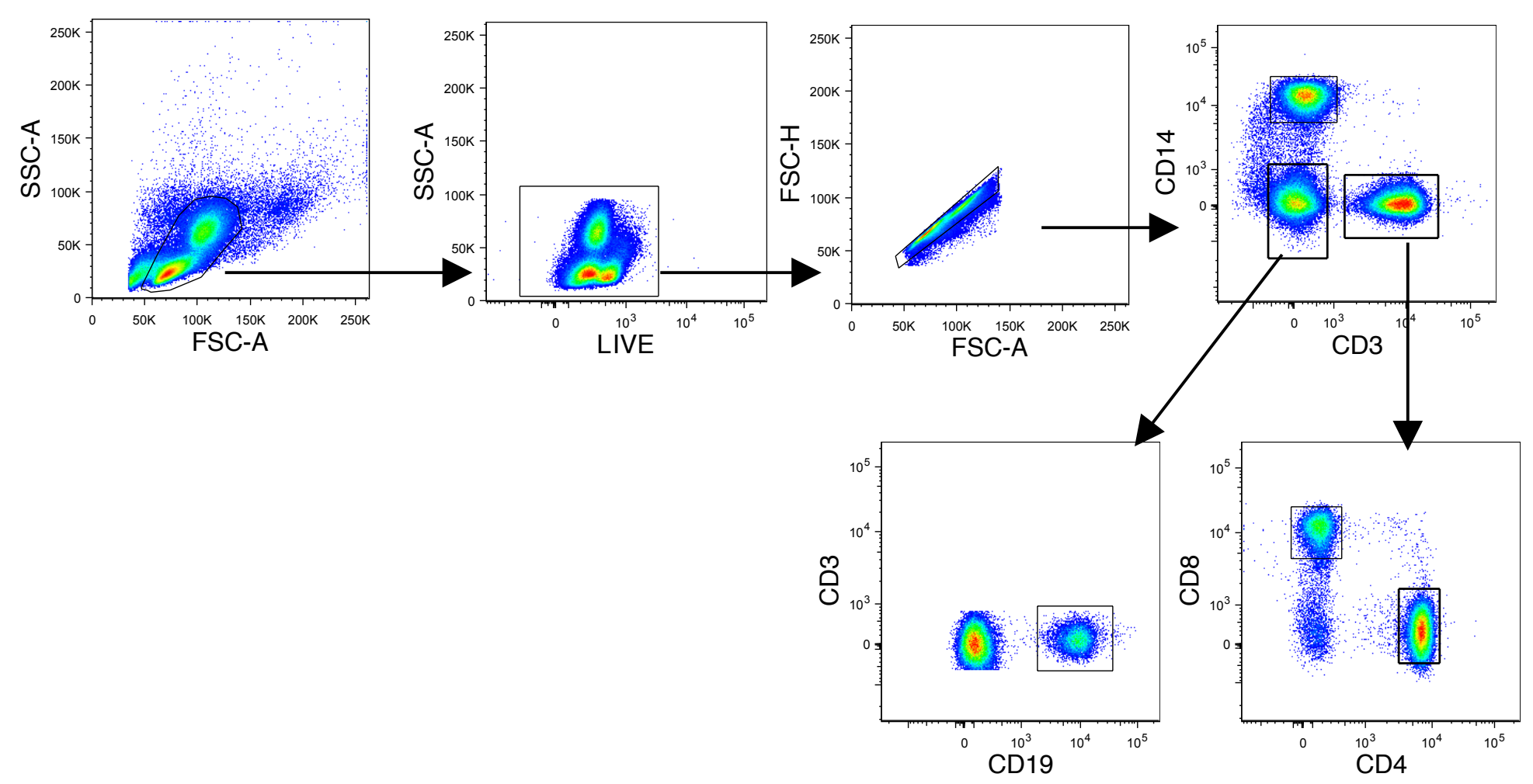

Neutrophil Gating Strategy
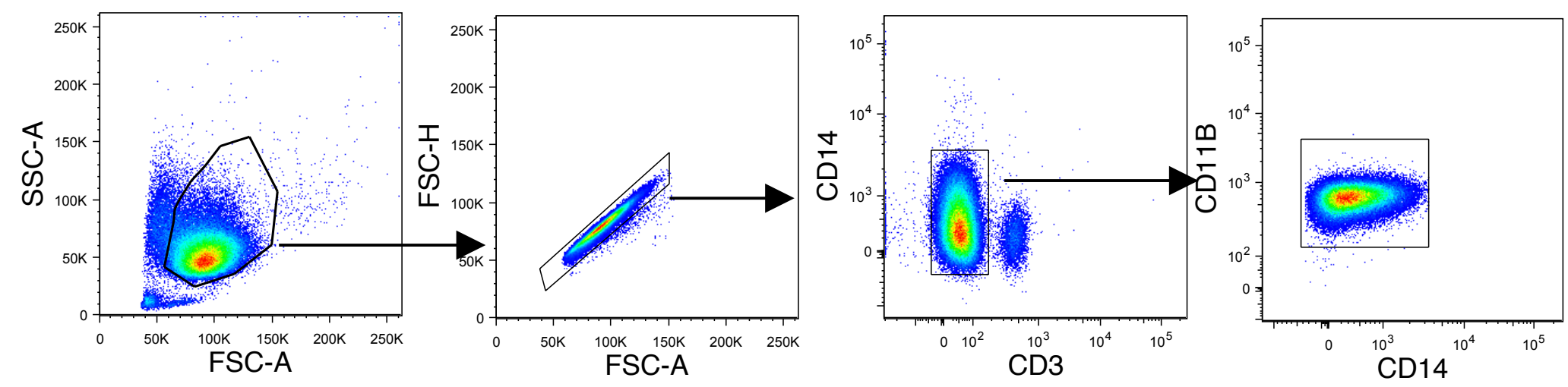

FAM129A
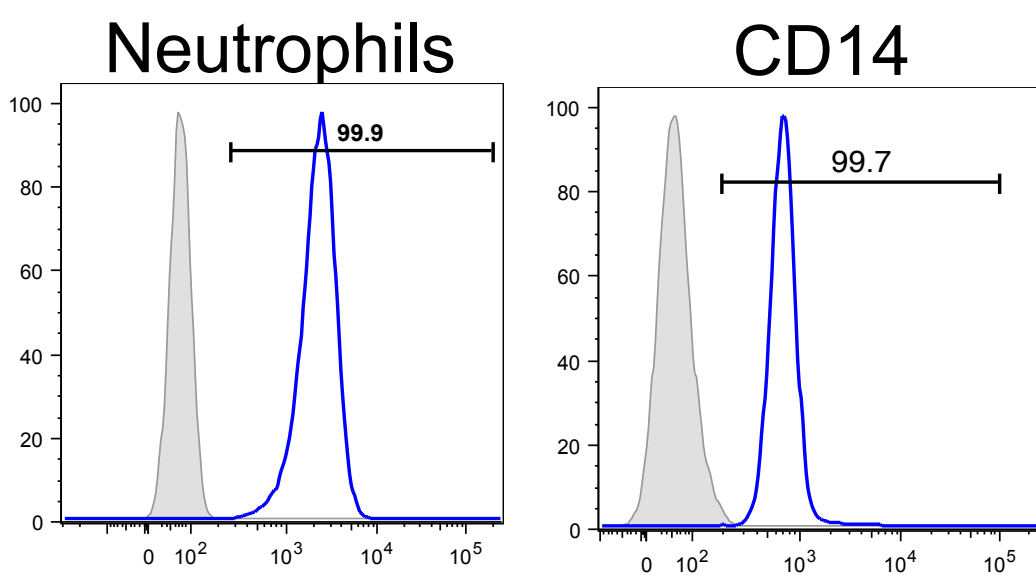

- Anti-FAM129A

- Isotype control

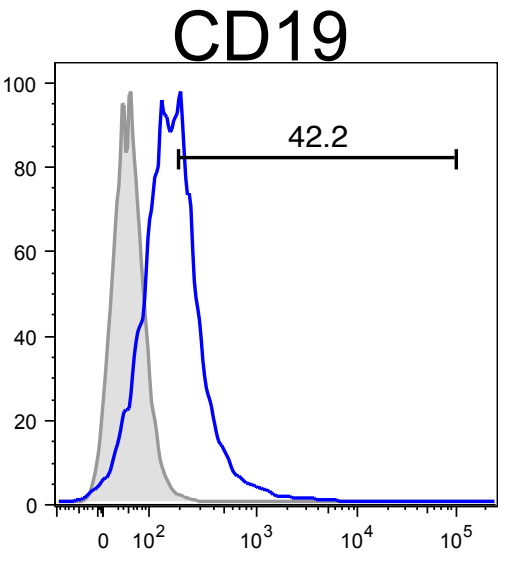

CD4

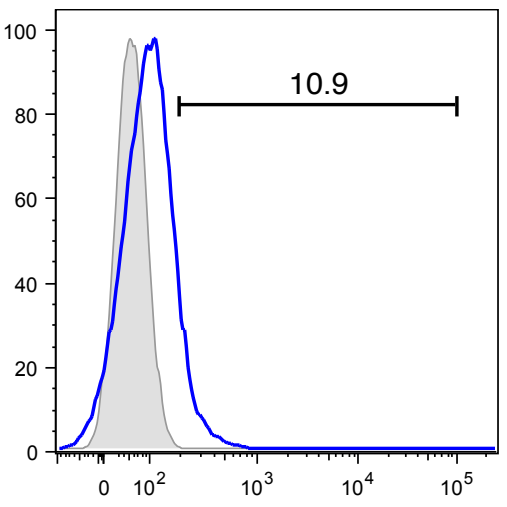

CD8

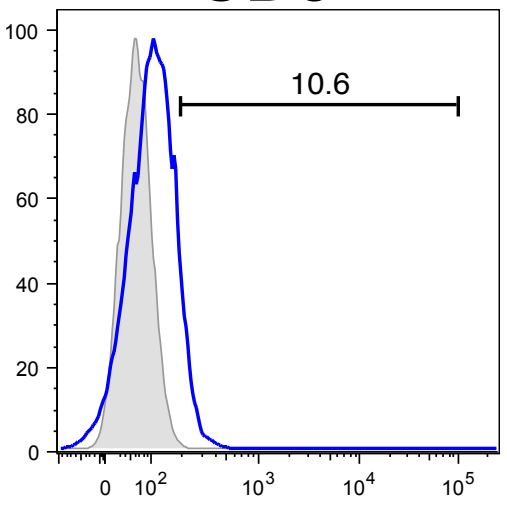

Figure 5 

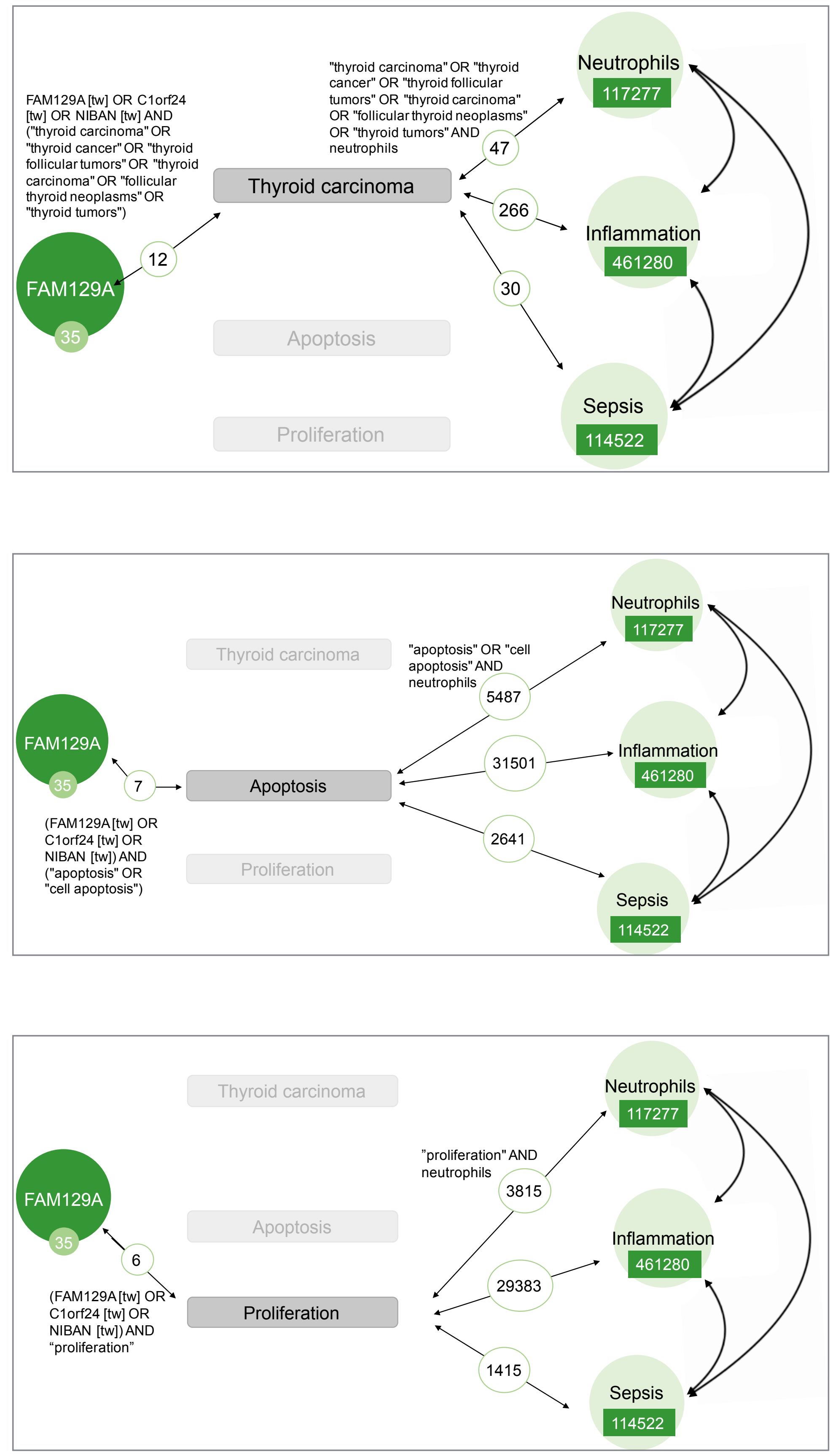